\title{
Exotic quantum phases and phase transitions in correlated matter *
}

\author{
Fabien Alet ${ }^{\mathrm{a}, \mathrm{b}}$, Aleksandra M. Walczak ${ }^{\mathrm{c}}$ and Matthew P. A. Fisher ${ }^{\mathrm{d}}$ \\ a Laboratoire de Physique Théorique, UMR 5152 of CNRS, Université Paul Sabatier, 31062 Toulouse, France \\ b Service de Physique Théorique, URA 2306 of CNRS, CEA Saclay, 91191 Gif sur Yvette, France \\ ${ }^{\mathrm{c}}$ Department of Physics and Center for Theoretical Biological Physics, University of California at San Diego, La Jolla, \\ California 92093, USA \\ d Kavli Institute for Theoretical Physics, University of California, Santa Barbara, California 93106, USA
}

\begin{abstract}
We present a pedagogical overview of recent theoretical work on unconventional quantum phases and quantum phase transitions in condensed matter systems. Strong correlations between electrons can lead to a breakdown of two traditional paradigms of solid state physics: Landau's theories of Fermi liquids and phase transitions. We discuss two resulting "exotic" states of matter: topological and critical spin liquids. These two quantum phases do not display any long-range order even at zero temperature. In each case, we show how a gauge theory description is useful to describe the new concepts of topological order, fractionalization and deconfinement of excitations which can be present in such spin liquids. We make brief connections, when possible, to experiments in which the corresponding physics can be probed. Finally, we review recent work on deconfined quantum critical points. The tone of these lecture notes is expository: focus is on gaining a physical picture and understanding, with technical details kept to a minimum.
\end{abstract}

Key words: Quantum Spin Liquids, Topological Order, Critical Spin Liquids, Deconfined Quantum Critical Points PACS: 71.10.-w, 71.10.Hf, 71.27.+a, 75.10.Jm

\section{Introduction}

Traditional condensed matter physics is shaped by two ideas that can be traced back to Landau: the Fermi liquid theory and the theory of phase transitions [1]. These principles have proven very fruitful and enabled great progress in describing simple metals, semiconductors and insulators. However starting from the experimental discovery of the

\footnotetext{
^ This paper was written by Fabien Alet and Aleksandra Walczak with equal contribution and in consultation with Matthew Fisher, based on his lectures and transparencies.
}

fractional quantum Hall effect [2], and before that theoretical predictions in $1 d$ quantum spin chains [3], it has become clear that a large number of phenomena observed in condensed matter materials lie beyond the description of these two fundamental paradigms. Such effects are observed in systems with strongly correlated electrons, which include for instance high $T_{c}$ superconductors [4], heavy fermion materials [5] or transition metal oxides [6]. In these systems the interactions between the electrons need to be considered explicitly and are primarily responsible for the observed phases of matter, which cannot be explained in a Fermi liquid 
picture. These new quantum mechanical phases are present at zero temperature, but their effects can also be felt at higher temperatures. Furthermore the phases of matter which emerge do not necessarily differ by symmetry, although quantum phase transitions between them are sometimes possible: this is not reconciliable within the Landau theory of phase transitions. Therefore the standard Landau paradigms do not apply and one must seek new approaches to understand the nature of these exotic phases.

These notes are based on the lectures entitled "Exotic quantum phases and phase transitions in correlated matter" given by one of us (M.P.A.F) at the FPSPXI summer school in Leuven. They are meant to be pedagogical and they also reflect the process of understanding of the scribes (F.A. and A.M.W.). The lecture notes can therefore be useful as a basic introduction to the topic. We have also tried to keep in the tone of these notes the lively spirit of the lectures.

The discussion will consider one of the simplest systems in which strongly correlated electron physics emerges, that of Mott insulators and will focus in particular on the example of spin liquid phases. Spin liquids are exotic phases that occur in quantum spin systems with odd number of spins $S=1 / 2$ per unit cell when no symmetries are broken at zero temperature; as such, they can be thought of as unusual quantum paramagnets. We start with a discussion in Sec. 2 of the situations where the interactions between electrons matter and how these strong correlations can break down the traditional solid state physics paradigms. In the following two sections, we present two known classes of systems where these strong interactions stabilize exotic phases: topological (Sec. 3) and critical or algebraic (Sec. 4) spin liquids. We then present in Sec. 5 recent work on an explicit example of a quantum phase transition which cannot be explained naturally in terms of a Landau theory, and we conclude in Sec. 6 .

\section{Breakdown of traditional paradigms}

\subsection{Fermi liquid theory and strong correlations}

Fermi liquid theory has been remarkably successful explaining why band theory works, even in systems with interacting electrons. Band theory basically assumes free electrons. Assuming a oneto-one correspondence between the states of the free system and those of the interacting one, the Fermi liquid theory shows that interactions essentially dress up the electrons on the Fermi surface into a "quasi-particle" with admittedly renormalized characteristics (effective mass etc.), but with retained independent properties - justifying band theory.

According to the resulting band theory, electrons have allowed energy levels (bands) separated by forbidden energy regions (gaps) in the presence of the ionic periodic potential. For temperatures smaller than needed to thermally excite an electron over the gap, the electrons cannot move around if the highest energy band is filled and consequently the material is an insulator, whereas if the highest band is partially filled the material is a metal. Effectively, band theory predicts that systems with an even number of electrons per unit cell are usually insulators and those with an odd number are always metals.

These predictions are correct if the bands are wide enough, as compared to the characteristic Coulomb interaction between electrons, such that the Fermi liquid assumption holds. This is usually the case for Fermi surfaces formed by electrons with an atomic $s$ or $p$ character. For materials with electrons at the Fermi energy from partially filled atomic $d$ or $f$ orbitals, the overlap of these orbitals on neighbouring atoms is usually much smaller, since the interatomic spacing is still determined by the $s$ and $p$ orbitals. This results in a smaller kinetic energy of hopping and therefore narrower bands. In such cases the electrons are said to be strongly correlated. It is worth stressing that the Coulomb energy has the same magnitude in either case - it is the kinetic energy of the electrons which differs between systems of strongly correlated electrons and those correctly described by Fermi liquid theory. 
The validity of Fermi liquid theory is determined by the ratio of Coulomb and kinetic energies.

Strong interactions lead to a correlated motion of electrons, and understanding its precise nature is the challenge faced by theories of strongly correlated electron systems. For larger magnitudes of Coulomb repulsion, the electrons are less likely to hop to a neighbouring site and they effectively selflocalize on a given lattice site. To be more explicit, the electrons can virtually hop to another site and back, however the materials are insulators and will not conduct. Such materials, with an odd number of electrons per unit cell, that do no conduct because of Coulomb repulsion are named Mott insulators, as opposed to band insulators which result from filled energy bands. In fact band theory, which ignores the correlation effects that determine the nature of these materials, predicts that they are metals. Examples of such materials are transition metal oxides (e.g. cuprates, manganites, chlorides) with partially filled $3 d$ and $4 d$ bands and rare earth and heavy fermion materials with partially filled $4 f$ and $5 f$ bands.

The simplest model which takes into account the correlation effects is the Hubbard model

$H=-t \sum_{\langle i j\rangle, \alpha}\left[c_{i, \alpha}^{\dagger} c_{j, \alpha}+h . c.\right]+U \sum_{i} n_{i, \uparrow} n_{i, \downarrow}$,

in which the inter-site hopping of the electrons $t$ competes with on-site repulsion $U$. Here $c_{i}^{\dagger}, c_{i}, n_{i}$ are respectively creation, annihilation and density operators of an electron at lattice site $i$ with spin index $\alpha$ which can take on up or down values $(\alpha=\uparrow$ $, \downarrow)$. The model considers one electron per cell on a lattice and $\langle i j\rangle$ denotes pairs of neighbouring sites $i$ and $j$. If $t / U$ is large, electrons are essentially free. In the other limit, $U / t \gg 1$, the electrons are site localized and the nature of the system is shaped by the residual spin physics.

Kinetic energy favours two neighboring sitelocalized electrons to have antialigned spins, as a hop to a neighbouring site with the same spin orientation is not allowed due to the Pauli exclusion principle. Therefore at half-filling and in the large repulsion limit, the most elementary model that captures the dominant interactions in Mott insulators is the $S=1 / 2$ Heisenberg Hamiltonian with antiferromagnetic exchange $J$
$H=J \sum_{\langle i j\rangle} \mathbf{S}_{\mathbf{i}} \cdot \mathbf{S}_{\mathbf{j}}$

Here $\mathbf{S}=\left(S^{x}, S^{y}, S^{z}\right)$ are $S=1 / 2$ operators, which (up to a constant) are equal to the Pauli matrices $\left(\sigma_{x}, \sigma_{y}, \sigma_{z}\right)$. The strength of the exchange constant is determined in terms of the original energy scales by a second order virtual hopping process: $J \sim t^{2} / U$.

Although these models are quite simple to write down, they prove very complicated to solve. The Hubbard model, as well as a majority of the quantum spin models, may not be solved analytically apart from a few special cases. Moreover, unlike in classical problems, numerical calculations are generally not helpful. The exponentially large size of the Hilbert space prevents an exact diagonalization of $H$ and the fermion sign problem renders Monte Carlo calculations inefficient. Since the fermion wavefunction changes sign under interchange of particles, calculating the partition function requires summing a large number of positive and negative terms, which results in large numerical error. Therefore to solve the problem one must try to deduce behaviours that might appear based on toy models and/or use basic physical notions such as universality. In classical systems, universal, system independent physics emerges when the correlation length becomes much larger than the interatomic spacing. The difference in the order of the magnitudes of the two characteristic lengths is responsible for a length scale separation and leads to system independent behaviour. In quantum system, the scale separation necessary for the existence of universal phenomena, is provided by the large characteristic energy scale of electrons in solids ( $t$ or $U$ in the Hubbard model), which is orders of magnitude larger than room temperature.

\subsection{Ordering in a Mott insulator}

Let us now focus on the behaviour at zero temperature of Mott insulators, which can be described by a spin Hamiltonian such as the Heisenberg model Eq. (2) and its variants.

At $T=0$, Mott insulating systems usually break symmetries and develop order. For exemple, the $2 d$ Heisenberg model on the square lattice develops 


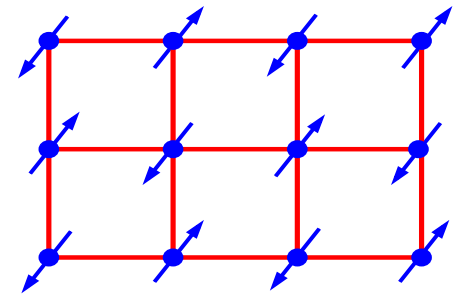

Fig. 1. The antiferromagnetic (or Néel) state on a square lattice.

(a)

$$
\longrightarrow=\frac{1}{\sqrt{2}}(\mid \uparrow \downarrow>-\| \uparrow>)
$$

(b)

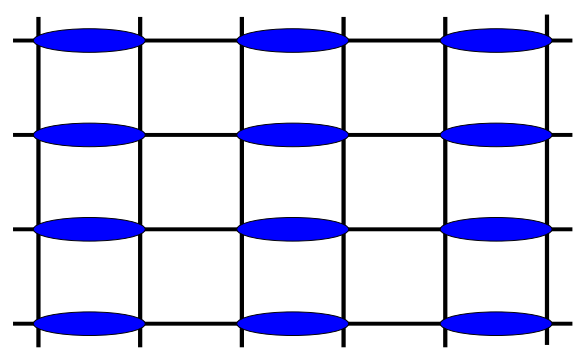

Fig. 2. (a) A valence bond (or a dimer) is a singlet pair formed by two neighbouring spins on a lattice. (b) The Valence Bond Solid (VBS) formed by crystalline ordering of these dimers.

antiferromagnetic (Néel) long range order associated with the breaking of spin rotation symmetry (see Fig. 1). Another possibility is that two neighbouring spins prefer to first pair themselves in a singlet $\frac{1}{\sqrt{2}}(|\uparrow \downarrow\rangle-|\downarrow \uparrow\rangle)$, forming a valence bond (a "dimer"). This is denoted in Fig. 2A by an oval. These dimers can then order amongst themselves, forming a valence bond solid (see Fig. 2B) which breaks translational symmetry. In both cases, these phases can be described by a local order parameter. Transitions in or out of these phases are then formulated within Landau's traditional theory of phase transitions, which expands the free energy in powers of this order parameter.

Our interest in the following sections lies elsewhere. We want to consider $S=1 / 2$ systems which do not break any symmetries when cooled to zero temperature - they do not possess long-range order. These systems, dubbed spin liquids, possess however some kind of exotic order, which cannot be understood in terms of a local order param- eter. A theorem recently proven by Hastings [7] guarantees the existence of exotic quantum ground states in Mott insulators with no broken symmetries. This theorem, a generalization of the LiebSchultz-Mattis theorem [8] to dimensions larger than 1 , states that for $S=1 / 2$ systems with one spin per unit cell on a two-dimensional lattice with periodic boundary conditions (on a torus), in the absence of symmetry breaking the ground state is separated from the first excited state by an energy gap that vanishes in the thermodynamic limit: $E_{1}-E_{0}<\frac{\ln (L)}{L}$, for a system of size $L$ by $L$. This means that in the thermodynamic limit, there cannot be a singly-degenerate quantum paramagnet with a finite energy gap (see Fig. 3a). In the following sections, we will describe two ways out of this situation and the exotic properties of the associated systems. Firstly we discuss in Sec. 3 topological spin liquids, where the disordered ground state is degenerated (see Fig. 3b) according to the topology of the system. These systems have an emergent gauge structure and admit excitations with fractional quantum numbers. In Sec. 4, we will describe critical or algebraic spin liquids, with gapless excitations above a ground-state that possesses power-law correlations (see Fig. 3c). Such systems behave without fine-tuning as if they were at a critical point.

\section{Topological spin liquids}

\subsection{Description of topological spin liquids}

As their name suggests, topologically ordered spin liquids have peculiar behaviour associated with the topology of the system. They have a degenerate ground state in the thermodynamic limit with a degeneracy that depends on the topology of the system. The ground state wavefunctions, associated with the degenerate states, cannot be distinguished by any local measurement but are globally distinct. These states also have an emergent gauge structure which supports gapped particle-like excitations, with quantum numbers that cannot be built up from electron quantum numbers. It is possible to partially classify such 
(b)

(a)

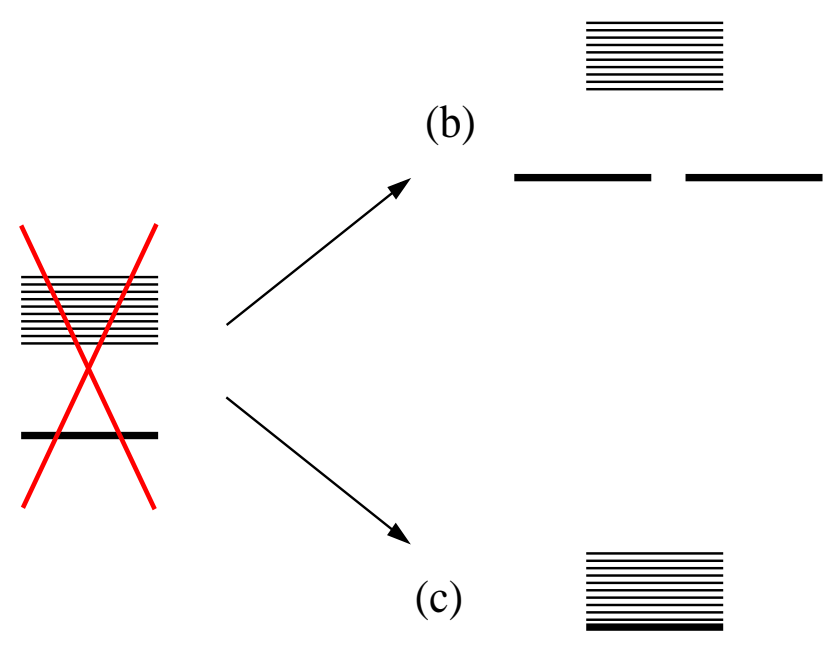

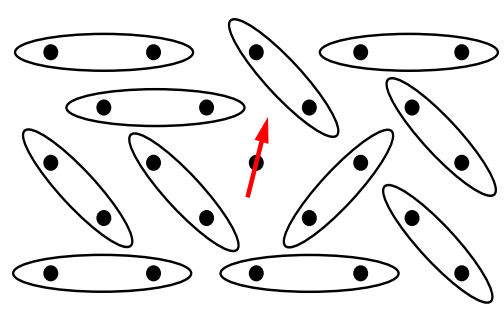

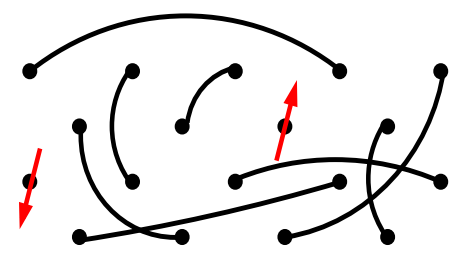

Fig. 3. Possible energy spectra (and associated real-space cartoon picture) of a spin liquid state with a disordered ground state in the thermodynamic limit. (a) For $S=1 / 2$ spin systems with odd number of spins per unit cell, the Hastings' theorem forbids non-degenerate ground state with a finite energy gap. (b) Topological spin liquids possess a multiply-degenerate ground-state (with a topology-dependent degeneracy) with a gap to all excitations in the bulk. The cartoon picture presents a RVB liquid state of dimers with a delocalized free spinon $S=1 / 2$ excitation. See Sec. 3. (c) Critical spin liquids possess a ground-state with power-law correlations and gapless excitations. In real space, critical spin liquids can typically be seen as valence bonds on many length scales. The excitations are spinons interacting with this valence-bond background. See Sec. 4 .

spin liquid phases in terms of the symmetry group of the emergent gauge theory. General discussions on topological order and its classification can be found in the book of Wen [21]. For simplicity, we will focus here on the simplest example of a topologically ordered system: a spin liquid with $Z_{2}$ topological order on a $2 D$ square lattice.

As proposed by P.W. Anderson [9], spin liquids can appear as a result of melting a valence bond solid. Consider a generalized Heisenberg model with not only nearest neighbours interactions

$H=\sum_{i j} J_{i j} \mathbf{S}_{\mathbf{i}} \cdot \mathbf{S}_{\mathbf{j}}$

with $J_{i j}>0$. As already mentioned, in some cases, two neighbouring spins will form, due to the antiferromagnetic interaction, a singlet pair (a valence bond). These singlets can possibly align to form the valence bond solid (VBS), depicted in Fig. 2b. The bonds between four neighbouring sites can also fluctuate quantum mechanically between two possible positions, as shown in Fig. 4a. These resonances between the bonds can lead to the melting (a)

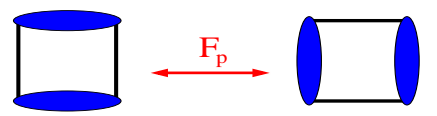

(b)

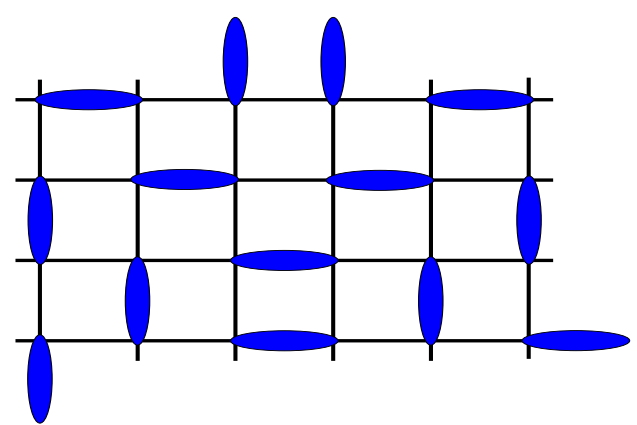

Fig. 4. (a) The two resonating states connected by quantum fluctuations on a plaquette. The flux operator $F_{p}$ flips the bonds between the two resonating states (see Sec. 3.2). (b) A Resonating Valence Bond (RVB) liquid is a disordered state of valence bonds.

of the solid to another state of matter with no longrange correlations of any kind: a resonating valence bond (RVB) liquid (Fig. 4b). The RVB liquid can be described using a wavefunction which is a su- 
perposition of many valence bond configurations.

What are the excitations of these two different phases? A valence bond may be broken, at an energy cost of the order of $J$, to create an $S=1$ (gapped) excitation in which the spins are aligned (see Fig. 5a). Let's try to separate these two $S=$ $1 / 2$ "spinons". The energy cost for this separation will grow linearly with separation in the VBS, because there will be a mismatch in dimer aligment all along the path separating the two spinons (see Fig. 5b). The spinons are therefore confined in the VBS, similarly to quarks in elementary particles. They cannot exist as finite energy excitations. However in the RVB liquid state, the region between the two spinons is not a defect line, as in the solid, therefore the energy costs stays finite with separation. The reorganization of the valence bonds to accomodate the extra spinon is local - or at least the energy cost is local. Therefore the spinons are deconfined in the liquid state (see the cartoon picture of Fig. 3b). Furthermore the spinons carry the $S=1 / 2$ spin of the electron, but do not carry any additional electrical charge. The charge density in the topological spin liquid is uniform and equal to one electron per cell. The existence of these fundamental excitations which cannot be described by electron quantum numbers is referred to spin-charge separation. Quantum numbers are said to be fractionalized, since the spin $S=1 / 2$ of the spinon is half the one of the conventional spin-flip excitation with $S=1$.

\subsection{Gauge theory formulation}

Due to their physical origin, the spin interactions in the Hamiltonian (3) are typically local. There is no explicit long-range interaction. How can we then understand the confinement/deconfinement properties of the spinons, which might be far apart? The natural framework for this is gauge theory. Let us now formulate the quantum dynamics of the valence bonds in such terms.

To formulate the problem in terms of a gauge theory, consider a "spin", on each lattice link, between two sites $i$ and $j$. This "spin" is described by a set of Pauli matrices $\sigma_{i j}$, the $x$ component of which takes on the value $\sigma_{i j}^{x}=1$ if there is no va- (a)

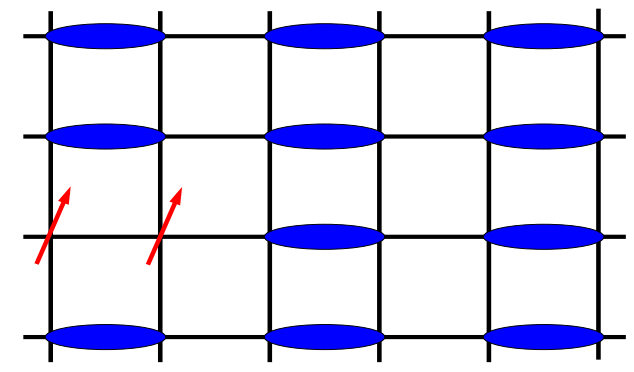

(b)

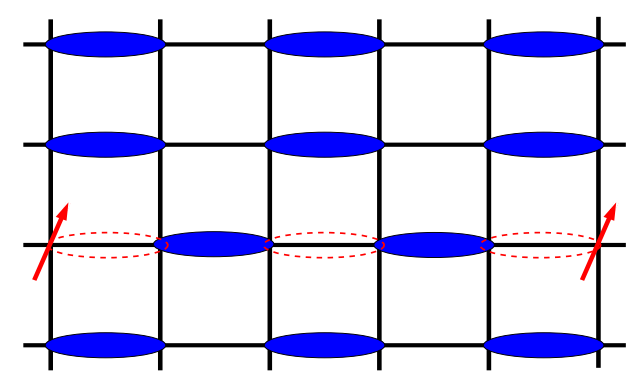

Fig. 5. (a) Breaking of a valence bond results in two spinon excitations. (b) In a VBS, two separated spinons have created a mismatch in the arrangement of dimers: the energy cost will be linear in the distance between spinons.

lence bond (no dimer) on the given link $(i, j)$ and $\sigma_{i j}^{x}=-1$ if there is one. We want to define an operator which, when acting on four spins on a plaquette, flips the bonds between the two resonating states, as depicted in Fig. 4a. This is easily done using operators that anticommute with each other, such as Pauli matrices. The bond flipping operator, called the plaquette flux operator, is defined on the four sites $i, j, k, l$ around the plaquette as $F_{p}=$ $\sigma_{i j}^{z} \sigma_{j k}^{z} \sigma_{k l}^{z} \sigma_{l i}^{z}$. The Hamiltonian which describes the resonating valence bonds is a sum over all plaquettes of the flux operators $H=-K \sum_{p(i j k l)} F_{p}$. Up to this point we have not taken into account the constraint that each spin can form only one singlet, i.e. only one valence bond comes from each site. Such a local constraint on the Hilbert space of this "quantum dimer model" may be encoded in a gauge charge $Q_{i}$ for each site $i$. The gauge charges are defined as the product of the bonds at a given site and we constrain them to be equal to $-1, Q_{i}=$ $\prod_{j=1}^{4} \sigma_{i j}^{x}=-1$. This constraint is still not sufficient, as the number of bonds at each site may still be equal to one or three. To get rid of the possibility of three bonds, we add a term to the Hamil- 
tonian, which minimizes the number of bonds at each site $-J \sigma_{i j}^{x}$, where $-J$ is the large energetic gain of having no dimer on a given link $(J>0)$. Therefore the full formulation of the problem is in terms of the Hamiltonian:

$H=-K \sum_{p(i j k l)} \sigma_{i j}^{z} \sigma_{j k}^{z} \sigma_{k l}^{z} \sigma_{l i}^{z}-J \sigma_{i j}^{x}$

with the constraint on the gauge charges $Q_{i}=$ $\prod_{j=1}^{4} \sigma_{i j}^{x}=-1$. This is equivalent to the formulation of a $Z_{2}$ gauge theory [19]. Note the, rather vague at this stage, analogy with the electrodynamics Hamiltonian $H=\mathbf{B}^{2}+\mathbf{E}^{2}$, supplemented by $\nabla \cdot \mathbf{E}=0$.

Since the gauge charge operator and the Hamiltonian commute $\left[Q_{i}, H\right]=0$, one can simultaneously diagonalize $Q_{i}$ and $H$. However not all eigenstates $H|E\rangle=E|E\rangle$ of the Hamiltonian are physically relevant states, as they do not satisfy the oddbond-per-site constraint. The projection operator $P=\prod_{i} \frac{1}{2}\left(1-Q_{i}\right)$ constructs a state $\tilde{E}$, that obeys the bond constraint, out of the eigenstates $E$ of the Hamiltonian. It is also worth noting that the Hamiltonian is invariant under a gauge transformation $\sigma_{i j}^{z} \rightarrow \epsilon_{i} \sigma_{i j}^{z} \epsilon_{j}$ with $\epsilon_{i}= \pm 1$. Note the analogy with the gauge transformation $\mathbf{A} \rightarrow \mathbf{A}+\nabla \phi$ in electromagnetism. The physical observables, the bond field $\sigma_{i j}^{x}$ (the "electric field" in the analogy) and the plaquette flip $F_{p}$ ("magnetic flux") are also gauge invariant. However the gauge field itself is not invariant under the gauge transformation. We ought to speak in our case of a gauge redudancy rather than gauge invariance, since the states related by a gauge transformation are physically equivalent. The total Hilbert space for a $N$ site square lattice model with $2 N$ links has $2^{2 N}$ states. Each gauge inequivalent class, however, has a redudancy of $2^{N}$. Therefore the number of physical distinct states is $\frac{2^{2 N}}{2^{N}}=2^{N}$, which correspond to the fluxes through the $N$ plaquettes $F_{p}= \pm 1$.

We can now gain insight about the confined VBS and deconfined RVB liquid phases of the dimer model from the known phase diagram of a $Z_{2}$ gauge theory, which is depicted in Fig. 6 in terms of the parameters $J$ and $K$. In the $J=0$ limit, the plaquette flux is fixed $F_{p} \approx 1$, as such a flux configuration minimizes the first term in the Hamiltonian

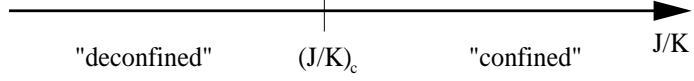

Fig. 6. Phase diagram of the $Z_{2}$ gauge theory model Eq. (4). For small values of $J$, the plaquette flux is fixed and the spinons are deconfined in the spin liquid phase. For $J \gg K$, the bond field is fixed, which corresponds to confined spinons in the VBS state.

Eq.(4). The conjugate bond field $\sigma_{i j}^{x}$, however, is strongly fluctuating. This corresponds to the resonating valence bonds in the deconfined spin liquid state. In the opposite limit, $J \gg K$, the bond field is fixed and the plaquette flux fluctuates, which describes the behaviour of the VBS. The dimer model was formulated assuming the energy cost $J$ of having a bond at a given link is large compared to $K$, to fulfill the one-bond-per-site constraint. From the phase diagram in Fig. 6, we see that for the quantum dimer model on a square lattice, this set of parameters corresponds to the confined state. In order for the system to be in the deconfined phase, one would have to relax this constraint on the value of $J$ or the gauge charge constraint. The latter scenario is realized, for example, for non-bipartite lattices, such as the triangular lattice for which a RVB liquid phase exists [10].

Many things are known about the $Z_{2}$ gauge theory, which is dual to the $2 D$ Ising model in a transverse field. In particular, we have a way to characterize the different phases through the Wilson loop operator $W_{L}$, defined as the product of the gauge operators $\sigma^{z}$ around a loop of size $L: W_{L}=$ $\prod_{i \in L} \sigma_{i}^{z}$. The expectation value of the operator for a loop of size $L$ decays as an exponential of the perimeter of the loop $\left\langle W_{L}>=e^{-c L}\right.$ in the deconfined phase and as the exponential of the area substanded by the loop $\left\langle W_{L}\right\rangle=e^{-c L^{2}}$ in the confined phase. We refer to Ref. [19] for an intuitive derivation of these two different behaviours.

\subsection{Analogy with electromagnetism, vison excitations and ground-state degeneracy}

The vaguely sketched analogy between the $Z_{2}$ gauge theory of spin liquids and electrodynamics may be further exploited. It can easily be shown, that the bond field and the plaquette flux do not commute whenever the bond surrounds the pla- 

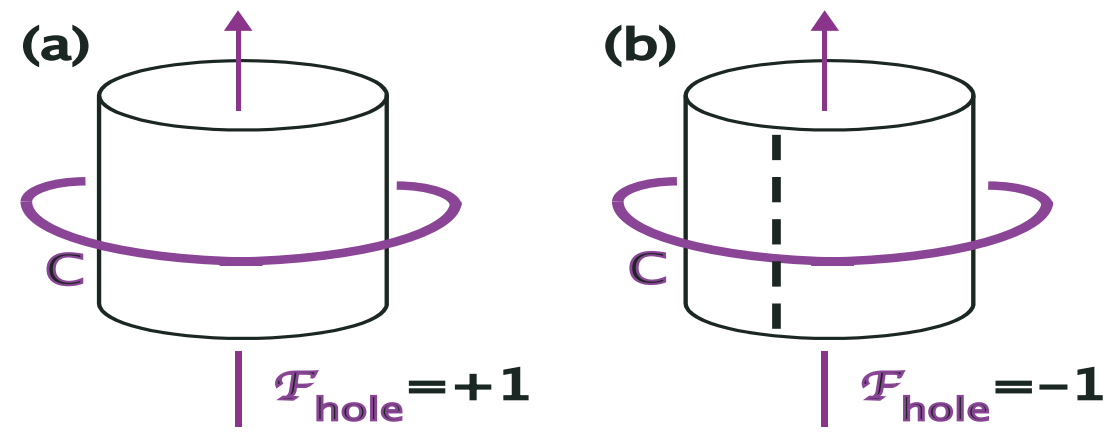

Fig. 7. The two degenerate states in the deconfined (RVB) phase on a cylindrical topology. (a) No vison is present. (b) A vison has threaded the cylinder. All the links that cross the dashed line have $\sigma_{i}^{z}=-1$ (whereas $\sigma_{i}^{z}=1$ for the rest of the system).

quette. Based on the Hamiltonian Eq.(4), we can identify the first with the electric field and the latter with the magnetic flux. Being non-commuting fields, the Heisenberg uncertainty principle applies and one field has to fluctuate if the other one is fixed.

In the deconfined spin liquid phase, the flux through all the plaquettes in the ground state is constant $\left(F_{p} \approx 1\right)$ and the bond field fluctuates a lot (the dimers are disordered), as argued above. If the flux through one plaquette is -1 , an excitation is created in the liquid, which has an energy cost of the order of $K$. This gapped excitation is called a vison and takes its name from an Ising vortex. Two visons on the same plaquette "annihilate" since the flux through the plaquette can only be \pm 1 - hence the reference to Ising. The vison is a non-magnetic $(S=0)$ excitation, of a different kind than the spinons described earlier. We are describing a liquid phase and both visons and spinons are finite energy excitations. How can we reconcile this with the Hastings theorem about the existence of low energy excitations, which tend to zero in the thermodynamic limit? The existence of the visons allows us to understand how this theorem may be satisfied. This theorem is fulfilled because the ground state is topologically degenerate, when the model is defined on nonsimply connected spaces such as a torus. In conventional paramagnets, the properties such as ground-state degeneracy are not affected in the thermodynamic limit by the choice of boundary conditions. This is not true anymore in a topological spin liquid.

To see this, let us take the cylinder topology, where a $2 d$ system has periodic boundary conditions in one direction and free in the other. In the deconfined phase, locally the flux through each plaquette is $F_{p} \approx 1$, but what about the hole in the cylinder? Consider the flux through any curve $C$, that encircles the cylinder $F_{\text {hole }}=\Pi_{i \in C} \sigma_{i}^{z}$ (Fig. 7). In the deconfined phase of the gauge theory, we can have both situations where there is a flux through the hole, or no flux through it. In the limit $K \rightarrow \infty$, a simple ground-state is obtained when all the link fields are positive $\sigma_{i}^{z}=1$. There is no vison present and $F_{\text {hole }}=1$ (Fig. 7 (a)). Now if we flip to $\sigma_{i}^{z}=-1$ all the dimers along a column of horizontal bonds that runs the length of the cylinder, we have a new state where $F_{\text {hole }}=-1$ and where a vison has "threaded the hole of the cylinder" (Fig. 7 (b)). On a finite system, this is an excited state when $J \neq 0$, but in the thermodynamic limit the energy cost of creating this line of defect tends to zero and this state also becomes a ground state. Hence the two-fold degeneracy of the ground state. The multiplicity of the degeneracy of the ground state therefore depends on the topology of the space of system; more specifically on its genus in our example. For example, the degeneracy is four-fold on a torus. Ref. [20] describes in great detail the possible interpretations of this degeneracy and its topological nature.

Moreover, from this example we see the hidden topological order is a global property of the whole 
system and cannot be seen on the local scale $\left(F_{p}=\right.$ 1 everywhere in the "bulk" for both degenerate ground states). It is easy to guess that it is impossible to deduce whether a vison has threaded or not the hole of the torus by looking locally at the dimers. Therefore one cannot distinguish between the different ground state wave functions by any local probe or order parameter; they are topologically distinct.

In the VBS phase, the bond (electric) field is fixed (the dimers order) and the magnetic flux fluctuates. This means that visons have proliferated, they are condensed in the VBS. A site with a spinon has no connecting valence bonds and since the gauge charges are defined in terms of the number of bonds in the system, such a spinon carries a gauge charge $Q_{i}=-1$, which can be thought of as an "electric" charge. These electric charges cannot propagate through the fluctuating "magnetic" fluxes. This is another way to see that the spinons are confined and no longer present as finite excitations in the valence bond solid phase.

With this electromagnetic analogy, we can also understand the nature of the interaction between the different excitations. A vison can be though of as a "magnetic" flux. Hence if a vison is taken around a spinon (the electric charge), analogously to the Aharonov-Bohm effect, it will result in a sign change in the wavefunction of the spinon $\Psi_{s} \rightarrow$ $-\Psi_{s}$. This may be thought of as a long-range "statistical" interaction mediated by the gauge field.

\subsection{Towards realistic models}

We have shown how by melting a VBS into a RVB liquid one obtains deconfined finite energy excitations - spinons. These can be described in terms of a $Z_{2}$ gauge theory. Exploiting the analogy with electromagnetism, one can identify another type of excitation - visons, which correspond to magnetic fluxes. By considering the topology of the space, on which the model is defined, one discovers the ground states are degenerate. Hence Hastings' theorem is satisfied, and exotic quantum ground states exist. Having identified the finite energy excitations, one can complete the electromagnetic analogy by considering the Aharonov-Bohm type interactions between the "electric" and "magnetic" charges.

Here we have only focused on phenomenological description of spin systems which can have a topological $Z_{2}$ spin liquid phase. Actually, there are already several models which can be shown (sometimes on rigorous grounds) to sustain $Z_{2}$ topological order. Without being exhaustive, one can mention quantum dimer models on non-bipartite lattices [10], Ising-like models with multiple spin interactions [11], rotor bosonic models [12] and more recently models Heisenberg-like spin models with original $S U(2)$ symmetry [13] or without [14]. We should also note that the $Z_{2}$ spin liquid is only the tip of the iceberg: there are many much more intricate topologically ordered phases possible, some with fractional and even non-Abelian statistics [15]. These phases are very promising candidates as possible realizations of quantum computers, as they could be topologically protected from decoherence effets [16]. We also have kept the discussion to $2 d$ systems, but this is not essential to obtain topological order. It is, however, necessary to obtain the fractional statistics and non-abelian properties.

Finally, we note that at this point theoretical models are sadly not very realistic. It remains a huge challenge to develop theoretical techniques to look for topological spin liquids in realistic models and find them in the laboratory. There is no experimental evidence, except for the fractional quantum Hall effect, as to the existence of topologically ordered phases. It was theoretically proposed [17] that topological order and visons could exist in high $T_{c}$ cuprates superconductors. Later experiments [18] disproved this statement. Nevertheless, these ideas and the proposed experimental set-up to detect visons could be useful to eventually experimentally engineer a real topological ordered phase, for example in a Josephson Junction array. The key ingredient is certainly to try to disorder the system as much as possible. 


\section{Algebraic or Critical Spin Liquids}

\subsection{Description of algebraic spin liquids}

Besides topological degeneracy, an alternate way in which an apparently featureless quantum paramagnet can remain "gapless" as required by the Hastings' theorem, is by having gapless bulk excitations - i.e. being a "critical" quantum phase. Such critical quantum ground states have powerlaw forms for correlation functions, but no true long-range order. The lack of a finite order parameter is necessary for a phase to be called a spin liquid. In some sense, these systems behave as if they were at a critical point without being tuned. A physical picture in terms of the spin degrees of freedom for such critical spin liquid phases can be visualized in terms of valence bonds between pairs of spins separated by arbitrary distances. The valence bond lengths follow a power-law distribution. These valence bonds quantum-mechanically fluctuate, which produces the continuum of excitations mentioned above. The spinons (unpaired spins), unlike in topological spin-liquids, are strongly interacting with this valence bond background and cannot be considered as free particles. A cartoon picture of a critical spin liquid is given in Fig. 3c.

Much less is known about critical spin liquids than topological spin liquids, as introduced in Sec. 3. Technically, the theoretical models involved are more complicated and even toy models are difficult to construct. Apart from the general considerations given in the preceding paragraph, there is no clear overall theoretical picture of all the generic features of critical spin liquids, although some progress has recently been made in this direction [22]. However, an advantage over topological spin liquids is that there are several experimental spin systems which are good candidates for being a critical spin liquid, and can thus be confronted with theory. We will describe one particular theoretical scenario which gives rise to a critical spin-liquid phase, which is consistent with current experiments on one promising compound. This example will let us identify some of the elements that are needed to form a critical spin-liquid.
4.2. Triangular lattice $S=1 / 2$ antiferromagnets

- Experimental evidence of spin liquid behaviour

As already mentioned, the key idea to obtain a spin liquid is to enhance the conditions that can supress magnetic order, namely using low spins $(S=$ $1 / 2$ or $S=1$ ), low dimensional systems, and geometric frustration, as in non bipartite-lattices [23]. The 1d Heisenberg chain is the simplest example of a critical system - it is much harder to find one in $2 \mathrm{~d}$. Our interest lies in $2 \mathrm{~d}$ systems, thus we will consider a spin 1/2 antiferromagnetic sytems on the triangular lattice. Numerical calculations have determined that the ground state of the isotropic Heisenberg model on the triangular lattice has long range coplanar magnetic order, with spins ordering in a 120 degrees fashion. This order is the same as for the classical ground state of the model, which results from the minimization of the frustrated interactions. However, in the $S=1 / 2$ quantum system, the magnetization is significantly reduced compared to the $S \rightarrow \infty$ classical limit, due to quantum fluctuations. This numerical result, along with recent experiments, gives one reason to believe that a spin liquid state is perhaps accessible in such triangular lattice antiferromagnets.

Currently, two experimental systems are likely candidates for spin liquid behaviour in the triangular $S=1 / 2$ antiferromagnet: an organic material $\kappa$ - $(\mathrm{ET})_{2} \mathrm{Cu}_{2}(\mathrm{CN})_{3}$ (Ref. [24]) and a transition metal material $\mathrm{Cs}_{2} \mathrm{CuCl}_{4}$ (Ref. [25]). We will focus the discusion on the latter system for which the $\mathrm{Cu}$ atoms have nine electrons on the $d$ shells and the one missing electron results in the $S=1 / 2$ moment. The experimental phase diagram for $\mathrm{Cs}_{2} \mathrm{CuCl}_{4}$ (Fig. 8), shows a long range magnetically ordered phase at very low temperatures and a "spin liquid" in an intervening temperature regime. At higher temperatures the spins on the $\mathrm{Cu}$ atoms fluctuate independently and the magnetic susceptibility obeys the traditional Curie-Weiss law. The planar triangular lattices in the $\mathrm{Cs}_{2} \mathrm{CuCl}_{4}$ crystal are stacked on top of one another, with a large interplanar distance. Therefore the in-plane interactions $J$ and $J^{\prime}$, as depicted in Fig. 9, are dominant. The magnetic long range ordered state is coplanar as predicted 

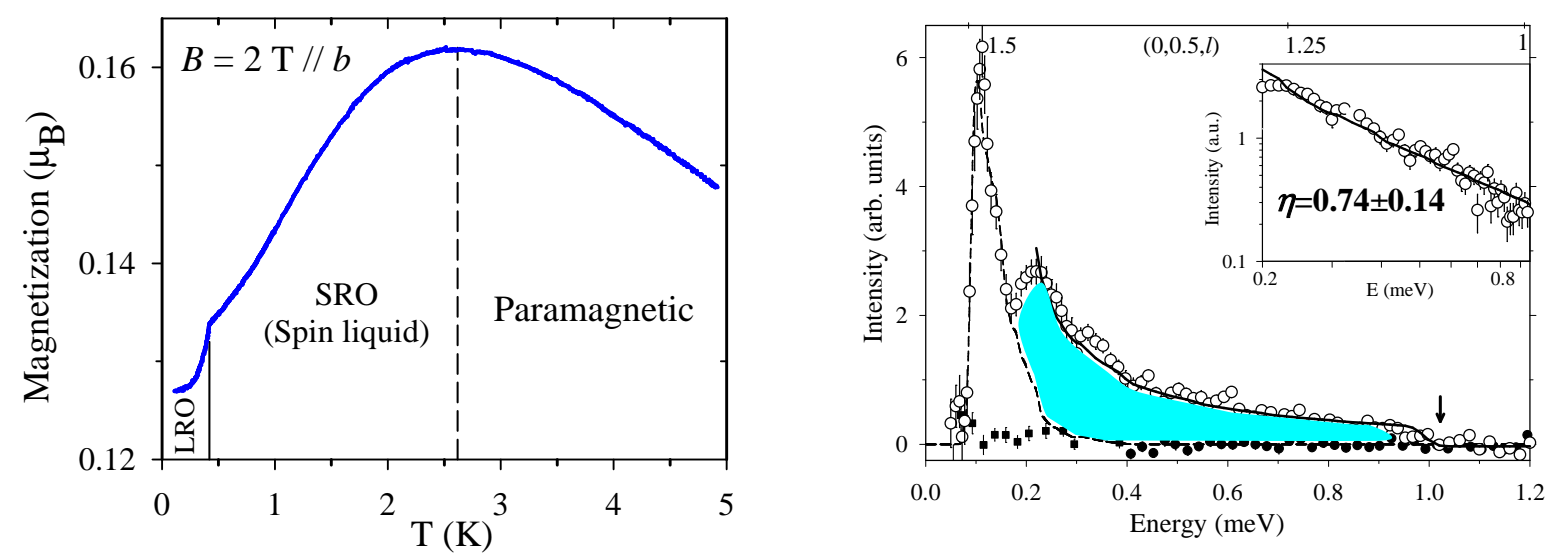

Fig. 8. Experimental results obtained on $\mathrm{Cs}_{2} \mathrm{CuCl}_{4}$. Left: Low field magnetization versus temperature (taken from Ref. [25]d). At the transition from the long-range ordered (LRO) state at low temperatures to the short-range ordered (SRO) spin liquid at intermediate temperatures the magnetization exhibits a cusp. The broad peak at the transition from the spin liquid to the paramagnet present at high temperatures is characteristic of short-range antiferromagnetic correlations. Right: Neutron scattering intensity as a function of energy transfer (taken from Ref. [25]b). The sharp peak at low energy is the signature of long-range ordering. The broad continuum at high energies can be fitted by a power-law (see inset) and is ascribed to the low-energy excitations of a critical spin liquid state.

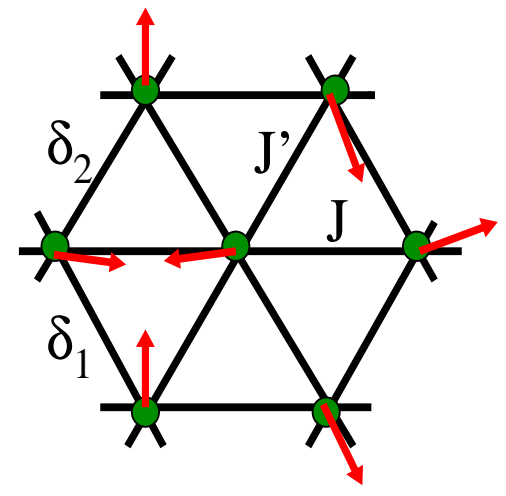

Fig. 9. The long-range coplanar ordered state adopted by the $S=1 / 2$ antiferromagnet $\mathrm{Cs}_{2} \mathrm{CuCl}_{4}$ (see Ref. [25]). The bond directions $\delta_{1}$ and $\delta_{2}$ and the interaction strengths $J$ and $J^{\prime}$ in the effective Hamiltonians Eq.(6) and Eq.(7) are also shown.

by numerical studies. Upon moving through the lattice the spins spiral in the plane. However, since the exchange interactions $J$ and $J^{\prime}$ differ in magnitude the wavelength of the spiraling spins is incommensurate with the lattice, resulting in the spin ordering represented in Fig. 9.

In $\mathrm{Cs}_{2} \mathrm{CuCl}_{4}$ the experimentally detected "spin liquid" only exists at finite temperature, whereas a theoretical spin liquid is well defined only at $T=0$.
Despite this drawback, there are two significant advantages of $\mathrm{Cs}_{2} \mathrm{CuCl}_{4}$ that compensate. Firstly, it is possible to grow large high purity single crystals, which may be used in neutron scattering. The main quantity which can be accessed in neutron scattering experiments is the magnetic structure factor, which is the Fourier transform of the spin-spin correlation function. At low temperatures, the experiments show magnetic Bragg peaks at wavevectors corresponding to the wavelength of magnetic ordering, which detect the spiral long-range ordered state (see Fig. 8). Inelastic neutron scattering experiments at higher temperatures, where the long range order is destroyed, can measure the strength of quantum fluctuations in the putative "spin liquid". Strikingly, these measurements show a power law dependence on energy transfer $\omega$ of the structure factor

$$
\left\langle S^{+}(\mathbf{q}, \omega) S^{-}(-\mathbf{q},-\omega)\right\rangle \sim \omega^{\eta-2}
$$

with $\eta \sim 0.75$ for wavevectors $\mathbf{q}$ chosen close to those of the Bragg peaks. This power-law feature is suggestive of a critical spin-liquid phase. The intensity of the scattered beam as a function of $\omega$ is plotted in Fig. 8b. Two features can be observed. Firstly, a sharp low-energy peak which shows up at low temperature is the signal of spin-waves as- 
sociated with the long-range order. This peak disappears for $T>T_{c}$. Secondly, a broad continuum scattering which extends up to high $\omega$ suggests many low-energy excitations. The number of these low-energy excitations increases as the aforementioned power law, as the transfer energy is lowered (see log-log scale in the inset of the figure).

The second advantage of $\mathrm{Cs}_{2} \mathrm{CuCl}_{4}$ is that the exchange interactions $J$ and $J^{\prime}$ are very small, of the order of a few Kelvins. Therefore one can experimentally measure the parameters of the effective Hamiltonian. These experiments are conducted in high magnetic fields, where the spins are forced to be polarized along the external field. This is possible because the Zeeman energies involved are of the order of a few Kelvins, corresponding to magnetic fields of a few Teslas, which can easily be reached in the laboratory. Using a neutron beam a single spin may be flipped and by measuring the energymomentum dispersion relation for this flipped spin, the values of the exchange interaction may be extracted. The Hamiltonian for a single layer has the form of nearest neighbour exchange interactions on the triangular lattice (see Fig. 9)

$$
\begin{aligned}
H_{0}=\sum_{\mathbf{r}} & {\left[J \mathbf{S}_{\mathbf{r}} \cdot \mathbf{S}_{\mathbf{r}+\delta_{1}+\delta_{2}}\right.} \\
& \left.+J^{\prime}\left(\mathbf{S}_{\mathbf{r}} \cdot \mathbf{S}_{\mathbf{r}+\delta_{1}}+\mathbf{S}_{\mathbf{r}} \cdot \mathbf{S}_{\mathbf{r}+\delta_{2}}\right)\right] .
\end{aligned}
$$

In addition there is a term due to spin-orbit coupling, called the Dzyaloshinskii-Moriya interaction. In most systems this term is small, but it may not be neglected here, since the exchange interactions are small. The full Hamiltonian therefore has the form $H=H_{0}+H_{D M}$, where the Dzyaloshinskii-Moriya interaction takes the form:

$H_{D M}=-\sum_{\mathbf{r}} \mathbf{D} \cdot \mathbf{S}_{\mathbf{r}} \times\left(\mathbf{S}_{\mathbf{r}+\delta_{1}}+\mathbf{S}_{\mathbf{r}+\delta_{2}}\right)$

where the experimentally measured parameters are $J \sim 4.3 \mathrm{~K}, J^{\prime} / J \sim 0.34$ and $|D| / J \sim 0.053$ (Ref. [25]c). The Dzyaloshinskii-Moriya interaction breaks the $S U(2)$ symmetry down to a $U(1)$ symmetry, called the "easy-plane" symmetry.

The model presented cannot be solved analytically or numerically except on very small lattices. Again due to the frustrated interactions, Monte Carlo calculations are plagued by the sign prob- lem. To gain an understanding of the physics in the particular phases, it is instructive to consider a simpler model, which has the same symmetries as the Hamiltonian in Eq. (6) and (7). The fact that the Dzyaloshinskii-Moriya interaction breaks the $S U(2)$ to $U(1)$ symmetry suggests to use an anisotropic exchange interaction $J_{i j}^{z}<J_{i j}$, leading to the following easy-plane spin model:

$$
H_{\mathrm{xy}}=\sum_{\langle i j\rangle}\left[J_{i j}\left(S_{i}^{x} S_{j}^{x}+S_{i}^{y} S_{j}^{y}\right)+J_{i j}^{z} S_{i}^{z} S_{j}^{z}\right],
$$

where the notation $J_{i j}$ reminds us that there are two different coupling strenghts $J$ and $J^{\prime}$ in the real material $\mathrm{Cs}_{2} \mathrm{CuCl}_{4}$.

\subsection{Chain of transformations: from spins to} bosons, from bosons to bosonic vortices and from bosonic to fermionic vortices

Even this simpler model is still hard to solve. The theoretical challenge is to find other variables, which correctly describe these spin systems. Here we present one of the proposed approaches [26], which allows one to make progress, based on various transformations. In the limit $J_{i j}^{z}=0$, the model in Eq. (8) is the quantum version of the $X Y$ model. In $2 d$, we know that the classical $X Y$ model can be equivalently described in terms of vortices. An analogous duality transformation in the quantum system will lead to the description of the system in terms of quantum vortices. A second transformation, which fermionizes the vortices, which are bosons, proves useful to make the system tractable. This change of variables, called the Chern-Simons transformation [27], is the $2 d$ generalization of the well-known Jordan-Wigner transform in $1 d$.

First, we conveniently describe spin $1 / 2$ objects in terms of hard-core bosons. A spin up on a given site corresponds to the site being occupied by a boson, whereas a spin down corresponds to an unoccupied site. Hard-core bosons means that there is at most one boson per site. Then the $x-x$ and $y-y$ spin interactions are mapped onto the hopping of bosons, which is described by creation $S_{i}^{+}=S_{i}^{x}+i S_{i}^{y}$ and annihilation operators $S_{i}^{-}=$ $S_{i}^{x}-i S_{i}^{y}$. On a bipartite lattice, the sign of spin in- 
teractions (ferromagnetic or antiferromagnetic) is irrelevant, since the sign of the hopping term can be easily removed with a simple unitary transform. But for non-bipartite lattices, such as the triangular lattice, antiferromagnetic interactions between the spins lead to a positive sign for the hopping term. Within a boson picture this does not make sense since the bosons energy must be lowered by a near-neighbor hop. In order for the kinetic energy to have the "correct" negative sign for the hopping strength, one needs to do a canonical transformation to include a fictitious flux of $\pi$ through the triangles of the lattice. When the boson hops around a triangular plaquette, its phase picks up a factor of $\pi$, reestablishing the sign due to antiferromagnetic interactions. This $\pi$ flux produces a fictitious magnetic field, in which the boson hopping takes place. The Hamiltonian Eq.(8) in terms of bosons then takes the following form

$H_{x y}=-\sum_{\langle i j\rangle}\left[\frac{J_{i j}}{2} S_{i}^{+} S_{j}^{-} e^{i A_{i j}^{0}}+\right.$ h.c. $\left.+J_{i j}^{z} S_{i}^{z} S_{j}^{z}\right]$,

where the last term is interpreted as a nearest neighbour density-density interaction between the bosons and where $\int_{\text {triangle }} \mathbf{A}^{0} \cdot \mathbf{d l}=\pi$.

We now proceed with the next transformation. Bosons (in magnetic fields) are often described in terms of vortices [28]. Let us now focus on this new object and its properties. To define a vortex, one can consider the phase of the boson creation operator $S_{i}^{+} \sim e^{i \phi}$ (or equivalently the spin direction in the XY plane). A vortex is present when the integral of the gradient of the phase $\phi$ around a loop gives $\int \nabla \phi \cdot \mathbf{d l}=2 \pi$. Depending on the configurations of the spins in the triangle, they may result in a vortex, associated with a vortex number $N=1$, or an antivortex, associated with a vortex number $N=0$, as shown in Fig. 10a.

Since the original spin problem is frustrated, the two spin configurations in Fig. 10a are energetically equivalent. Therefore there are as many triangular plaquettes with vortices as with antivortices; the dual vortices are at half-filling.

In this description, the vortices live on the honeycomb lattice, which is dual to the triangular lattice. This is easy to see, by connecting sites at the centers of plaquettes of the triangular lattice - (a)

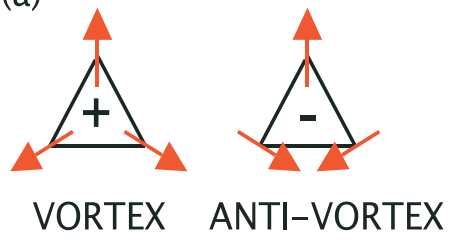

(b)

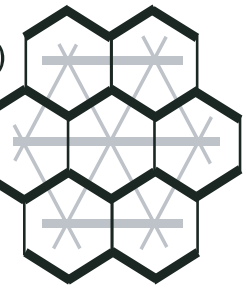

Fig. 10. (a) A pictorial representation of vortices and antivortices. (b) The honeycomb lattice is dual to the triangular lattice.

see Fig. 10b. The duality transformation between bosons (the original spins 1/2) and vortices introduces a gauge field a sitting on the links of the dual lattice. This gauge field will encode the longrange interaction between the vortices. This exact mapping relates, for example, the $z$ component of the spin to the field: $S^{z}+1 / 2=\frac{1}{2 \pi}|\nabla \times \mathbf{a}|$. This indicates that a vortex hopping around a dual plaquette will pick up a $\pi$ phase factor. This is easy to understand as a vortex hopping around a plaquette of the honeycomb lattice will encircle one spin $1 / 2$, and thus "feel" its average flux. Since on average $\left\langle S^{z}\right\rangle=0$ (the original spins are not in a magnetic field), the vortices experience an average background magnetic field of flux $\int d^{2} \mathbf{r} \nabla \times \mathbf{a}^{\mathbf{0}}=$ $\pi$. $\mathbf{a}^{\mathbf{0}}$ is the static part of the gauge field, precisely designed to account for the fact that the original spins can take only the values $S^{z}= \pm 1 / 2$.

The gauge field actually mediates a long-range Coulomb, or "electromagnetic", interaction between the vortices. In $2 d$, the Coulomb potential is logarithmic. The Hamiltonian in the vortex representation, where $b^{\dagger}$ and $b$ are the vortex creation and annihilation operators, reads:

$$
\begin{aligned}
H=-\sum_{\langle i j\rangle} & \left(t_{i j} b_{i}^{\dagger} b_{j} e^{\left.i a_{i j}+a_{i j}^{0}\right)}+h . c .\right) \\
& +\sum_{i j}\left(N_{i}-1 / 2\right) V_{i j}\left(N_{j}-1 / 2\right) \\
& +U \sum_{r}(\nabla \times \mathbf{a})_{r}^{2} .
\end{aligned}
$$

The first term is the kinetic energy of the vortices, moving on a honeycomb lattice in a field 
with static component satisfying $\left(\nabla \times \mathbf{a}^{\mathbf{0}}\right)_{r}=\pi$ for all sites $r$ of the triangular lattice. The second term is the Coulomb interaction between vortices with $V_{i j} \sim \ln (|i-j|)$. In this term we clearly see the half-filled natures of the vortices, as $N_{i}=b_{i}^{\dagger} b_{i}$ is the vortex number operator. The final term similar to a magnetic energy term - accounts for the dynamics of the gauge field. We refer again to Ref. [26,28] for a rigorous construction of the dual vortex hamiltonian.

This type of boson/vortex transformation is especially useful when the dual vortex variables are at integer filling. Here, however, due to the original spin frustration, the vortices are located at halffilling, since the states with filling fraction of zero or one are equally likely. The dual vortex representation thus appears as intractable as the original spin model. However, further progress can be made by performing yet another transformation, "transmuting" the bosonic vortices into fermionic vortices. The main advantage of this change of variables, is that the interacting boson field may be described using a non-interacting fermion field, since fermion statistics have Pauli exclusion built in. This makes the model mathematically easier. However, there is a price to pay. The fermion wavefunction changes sign upon exchanging particles, whereas the boson function does not. The transformation must therefore correct for this sign change. This Chern-Simons transformation [27] may be intuitively understood, as attaching a fictitious $2 \pi$ flux tube to a fermion vortex in order to represent the bosonic vortex. So if we consider the exchange interaction between two fermionic vortices, due to the Aharonov-Bohm effect, encircling one particle half way around the other results in a $e^{i \pi}$ flux from the interaction of the fermion with the flux tube of the other particle, which cancels the negative sign from fermion statistics.

Now let's look at a static picture of the fluxes attached to these fermions, which also live on the dual lattice. We can use a convention where each fermion shares on average its $2 \pi$ flux amongst the three honeycomb plaquettes to which it belongs. Since on average, each honeycomb plaquette has three vortices, as the vortices are at half-filling, it is therefore pierced by a $2 \pi$ flux. This is equivalent to a zero flux and therefore irrelevant for the behaviour of the fermions. Of course, this is a crude picture, but it is valid in the mean field approximation where we ignore flux fluctuations around their average. Therefore, the fluxes associated with the Cherns-Simon transformation cancel to zero when we smear out these fluxes in a mean field fashion. We note that any mean-field approximation is of course crude, but here it is actually expected to be rather good, since the interactions between the vortices tend to supress their density fluctuations, and therefore the flux fluctuations in the ChernsSimon language.

So now in the mean-field picture we are left with free fermions. However, we have to remember that these fermions are also coupled to the original gauge field $\mathbf{a}$, as were the vortices. In mean-field theory, we only consider the static contribution from $\mathbf{a}^{\mathbf{0}}$, and we are thus describing a system of free fermions on the honeycomb lattice with a background $\pi$ flux. This is a one-particle problem and it is easily solved. The corresponding Fermi "surface" consists of only four points, with Dirac-like (gapless) dispersions around each one.

The non-interacting limit we are currently describing, is a good starting point to now reintroduce the gauge fluctuations we supressed in the mean-field approximation. To do this, we now describe the behaviour of the low energy particlehole excitations around these four Fermi points in terms of a Dirac theory for fermionized vortices. There are four flavours of Dirac particles, which correspond to the four Fermi points. These fermionic vortices interact logarithmically, which is accounted for by a coupling to the gauge field a. Additionally, they are coupled to the ChernsSimon gauge field $\mathbf{A}_{\mathbf{C S}}$ due to the flux tube. It can be argued [26] that the field due to the flux tubes fluctuates much more than the field due to the Coulomb interactions between vortices and that at long length scales the Chern-Simons field can be ignored. This may also be seen explicitly by defining an auxilary field and integrating out the field due to the fictitious flux tubes. In fact, the logarithmic interaction in $2 d$ is so strong that it dominates over the statistics encoded by the Cherns-Simon flux tubes. In this respect the description in terms of fermions and bosons is anal- 
ogous. We invite the interested reader to consult Ref. [26] for a more detailed discussion.

The final formulation of the critical spin liquid in terms of $N=4$ flavours of Dirac particles, which interact by a logarithmic potential in $2 d$ is in terms of a QED (Quantum Electrodynamics) Lagrangian in $2+1$ dimensions

$$
L_{Q E D 3}=\overline{\psi_{a}} \gamma^{\mu}\left(\partial_{\mu}-i \tilde{a_{\mu}}\right) \psi_{a}+\frac{1}{2 e^{2}}(\nabla \times \tilde{\mathbf{a}})^{2},
$$

where $\psi_{a}$ is the spinor associated to the Dirac fermion flavour $a \in\{1,2,3,4\}, \gamma^{\mu}$ are Dirac gamma matrices in direction $\mu \in\{x, y, z\}$ and $\tilde{\mathbf{a}}=\mathbf{a}+\mathbf{A}_{\mathbf{C S}}$ is the auxiliary field used in the integration of the Cherns-Simon field (the sums over double indices are implicit). Strictly speaking, there should be an additional $L_{4 f}$ four-fermion term that originates from short-range interactions between the vortices that we have not taken into account explicitly, but that can always be present in the microscopic model. It is known that such terms are irrelevant in the limit of an infinite fermion flavour number $N \rightarrow \infty$ and for all $N>$ $N_{c}$, where the exact value of $N_{c}$ is not known.

Assuming $N_{c}<4$, we can safely ignore this term. Now, we can finally import the results from QED, which indicate that Eq. 11 describes a critical theory with gapless excitations. Thus as a result of these consecutive changes of variables, from spins to Dirac particles, this approach describes the much desired critical spin liquid phase, without fine tuning of variables and no free particles. This specific spin liquid is dubbed an "algebraic vortex liquid" [26].

In this section, we have tried to describe the different steps of the construction that lead to the algebraic vortex liquid. We will now only mention some of the properties of this type of spin liquid. The critical phase not only does not break any symmetries, but a $S U(4)$ symmetry emerges. Above all, the theory is able to make predictions, which may be compared with experiment. The structure factor is predicted to have power law correlations around five distinct momenta with the same exponent for each wavevector. Two of these momenta correspond to the experimentally measured magnetic long range order wave vectors. To make a further comparison with $\mathrm{Cs}_{2} \mathrm{CuCl}_{4}$, the Dzyaloshinskii-Moriya interaction be incorporated, and in the Dirac theory corresponds to a "mass" term which destabilizes the algebraic vortex liquid driving it into an incommensurate spiral state at low enough temperature: this is exactly what is observed in the experimental phase diagram of $\mathrm{Cs}_{2} \mathrm{CuCl}_{4}$ (see Fig. 8a).

The proposed algebraic vortex liquid state is not the only existing scenario to describe the physics of $\mathrm{Cs}_{2} \mathrm{CuCl}_{4}$. There have been recent theoretical proposals such as a quasi-1d approach [29], an algebraic spin liquid state with fermionic spinons and a $U(1)$ compact gauge field [30] or the proximity of a quantum critical point between a $Z_{2}$ spin liquid and a spiral phase [31].

We would finally like to emphasize that through the example described in this section, we can see that, like in the topological spin liquid case, the natural description of these phases is in terms of gauge fields. The gauge fields that emerge for critical spin liquids, however, have different symmetries (here $U(1))$ than the $Z_{2}$ symmetry for the topological spin liquids of Sec. 3. We will further exploit gauge theories in the next section.

\section{Deconfined quantum critical points}

We will now turn from the description of exotic phases of matter discussed in the two previous sections, to an exotic critical point separating two conventional states of matter. We will focus on the quantum phase transition between a Néel state, which breaks spin rotational symmetry, and a Valence Bond Solid state, which breaks translational symmetry, depicted in Fig. 1 and 2b. We will show that, contrary to general expectations of the Landau theory of phase transitions, a direct second order transition is possible. It is a continuous phase transition between two ordered states with different symmetries. We will find that, right at the critical point, the spinons we encountered in the two previous sections are essentially deconfined, whereas they are altogether absent in both surrounding phases. The description of this so-called "deconfined quantum critical point", will also be in terms of a gauge theory, namely a non-compact 


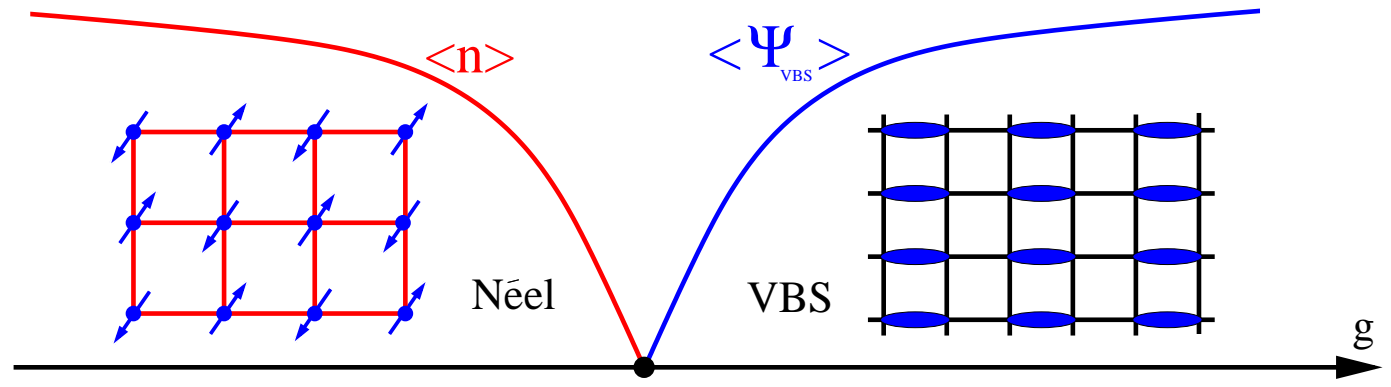

Fig. 11. The $T=0$ transition (driven by an external parameter $g$ ) between a Néel antiferromagnetic order and a Valence Bond Solid for spins $S=1 / 2$ on the square lattice. The transition is argued to be second-order [32] with both Néel and VBS order parameters vanishing continuously at the critical point.

$C P^{1}$ theory in this case.

\subsection{Formulation of the problem: the transition and standard Landau theory}

We want to describe $2 d$ quantum $S=1 / 2$ spin systems with one spin per unit cell on the square lattice and the quantum phase transition, i.e. a transition at zero temperature driven by an external parameter, between a Néel state and a VBS state (see Fig. 11). We do not explicitely mention a specific form of the microscopic interaction strength $g$ that drives the transition, which could be longer-range couplings like in Hamiltonian Eq. (3), or maybe a ring-exchange term, but rather focus on the symmetries of the associated phases.

If instead of a VBS state a simple paramagnet with no symmetry breaking existed on the righthand side of Fig. 11, it would be natural to describe the transition within a standard Landau approach. That is, we would identify a local order parameter, the staggered magnetization $\mathbf{n}_{i}=$ $(-1)^{\mathbf{r}_{i}} \mathbf{S}_{i}$, coarse-grain it to the continuum $\mathbf{n}(\mathbf{r})$ and expand the free energy or Lagrangian in gradients or powers of $\mathbf{n}(\mathbf{r})$ allowed by the symmetries such as $L(\mathbf{n})=|\nabla \mathbf{n}|^{2}+r|\mathbf{n}|^{2}+u|\mathbf{n}|^{4}+\ldots$ Depending on the precise value of the parameters, we would get either the Néel state $(r<0)$ or the paramagnet $(r>0)$, and the transition at $r=0$. Here the main problem is that the plain paramagnet does not exist in $2 \mathrm{D}$ at $T=0$, according to Hastings' theorem. It is thus natural to consider something else than the paramagnet, more specifically the VBS. In the case of a transition between two different broken symmetry phases, the Landau theory would expand the Lagrangian in terms of both the Néel order parameter $\mathbf{n}(\mathbf{r})$ and the VBS order parameter $\psi_{\text {VBS }}$ with possible cross terms.

For a phase transition between states with different broken symmetries, the resulting Landau theory predicts one of the four possible scenarios: (i) a direct first order phase transition, (ii) an intermediate coexistence phase, between Néel and VBS orders in our case, (iii) an intermediate phase with no order at all, (iv) a second order transition in the case of a multi-critical point. Scenario (iii) can be excluded using Hastings' theorem. Scenario (iv) is very unlikely, because it is not generic and it implies a fine-tuning of some parameter. Scenarios (i) and (ii) cannot be ruled out on general grounds, but both exclude a direct continuous (second-order) transition.

In contradiction to this standard Landau analysis, recent work [32] shows that in the specific case of a Néel-VBS transition on the square lattice, the transition can generically be second order without any fine-tuning, due to subtle quantum interference effets that invalide the Landau analysis. The crucial elements responsible for a continuous transition are topological objects called hedgehogs. The resulting field theory is a non-compact gauge theory. In what follows, we review in a simplified way the approach of Ref. [32].

\subsection{Néel order, skyrmions and hedgehogs}

To gain insight into such a Néel-VBS transition, let us first consider the Néel state, and its topologi- 


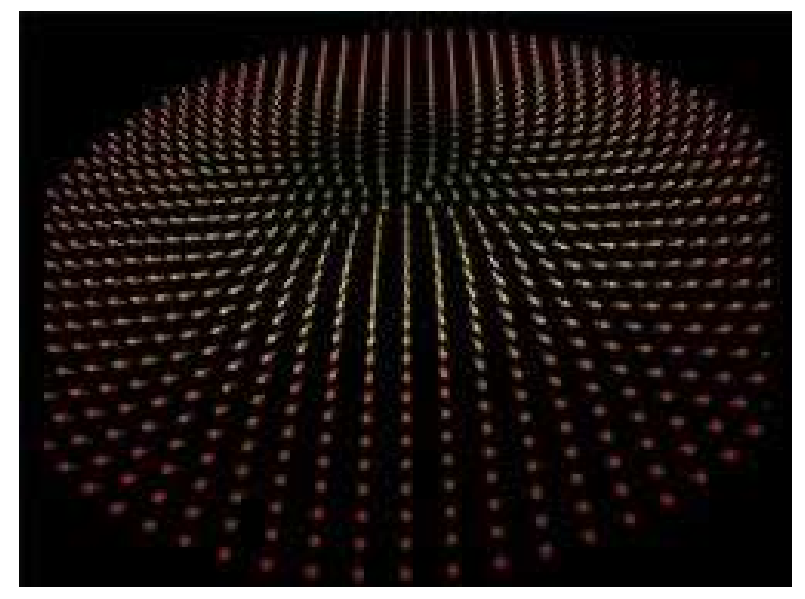

Fig. 12. Real-space representation of a skyrmion in the Néel field $\tilde{\mathbf{n}}$ at a given timeslice. Spins in the center are pointing down whereas spins on the boundaries point up. The charge of this skyrmion is $Q=+1$.

cal defects. Since we are considering a $2 d$ quantum system at $T=0$, the Néel vector lives in $(2+1)$ dimensions, where the extra-dimension can be interpreted as imaginary time in the standard path integral formalism. We shall denote the space-time coordinates as $x_{\mu}$ with $\mu \in\{x, y, \tau\}$. Consider smooth configurations of the staggered magnetization $\mathbf{n}\left(x_{\mu}\right)=(-1)^{x+y} \mathbf{S}\left(x_{\mu}\right)$, i.e. $\mathbf{n}\left(x_{\mu}\right)$ is slowly varying and is of constant amplitude. Let's define a unit length Néel vector $\tilde{\mathbf{n}}\left(x_{\mu}\right)=\mathbf{n}\left(x_{\mu}\right) /\left|\mathbf{n}\left(x_{\mu}\right)\right|$. For the Néel ground states, all the $\mathbf{n}\left(x_{\mu}\right)$ are pointing in the same direction, but there exist configurations which admit a topological defect called a skyrmion. A skyrmion is represented in Fig. 12, in which the Néel vector at the center of the "sample" points down whereas it points up at the borders. Mathematically, the skyrmion number at a given time slice can be defined as an integer topological number

$Q=\frac{1}{4 \pi} \int d x d y\left(\tilde{\mathbf{n}} \cdot \partial_{x} \tilde{\mathbf{n}} \times \partial_{y} \tilde{\mathbf{n}}\right)$

sometimes called an integer charge. If the spins were living in the continuum, this number would be a conserved quantity in time. However, on a lattice, this number can change from one time slice to the other. Such a space-time event where the skyrmion number $Q$ changes by \pm 1 is called a hedgehog (see Fig. 13a). The choice of this name hopefully be- comes apparent upon inspecting Fig. 13b, where a hedgehog is represented. We can clearly see a singular point from which all the $\tilde{\mathbf{n}}$ are pointing outwards, like quills on a hedgehog. A hedgehog is the term used for a configuration of unit Néel vectors, which is singular at one space-time point, but smooth elsewhere. What is the role of these topological defects? In a Néel state, hedgehogs are very costly energetically and are therefore absent. Deep within an ordinary paramagnet, the spins fluctuate essentially independently of one another. In this case there is no energetic reason why hedgehog events should be suppressed: we say that the hedgehogs proliferate.

To see what happens to these hedgehogs on the VBS side and at the critical point, it is useful to consider the non-linear sigma model description of the Néel state [33] with the following action:

$S_{\sigma}=\frac{1}{2 g} \int d x_{\mu}\left|\partial_{\mu} \tilde{\mathbf{n}}\right|^{2}+S_{\text {Berry }}$

where the first term accounts for the slow variations of $\tilde{\mathbf{n}}, g$ is some "stiffness" constant, and the second term is a Berry phase term which accounts for the quantum nature of the $S=1 / 2$ spins. Now let's see the effects of hedgehogs on such a formulation by expanding the partition function $Z=$ $\int d \tilde{\mathbf{n}} \exp \left(-S_{\sigma}\right)$ in powers of the fugacity $\lambda$ of such events: 


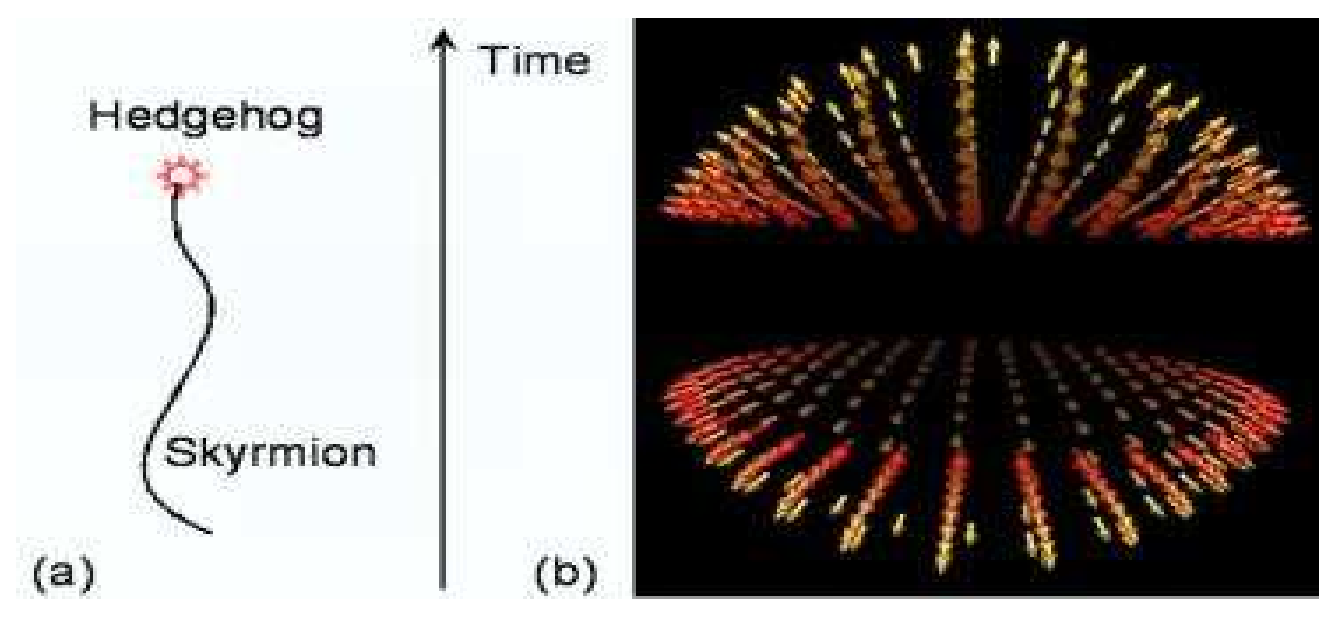

Fig. 13. (a) The skyrmionic charge is suddenly changed at a hedgehog event in space time. (b) Real-space representation of a hedgehog event (the two spin configurations represent different time slices). A hedgehog corresponds to a singular configuration of $\tilde{\mathbf{n}}\left(x_{\mu}\right)$ at one space-time point where the skyrmionic charge changes. All spins are pointing outwards of the hedgehog.

$$
\begin{aligned}
Z=Z_{0} & +\int_{x_{\mu}^{1}} \lambda\left(x_{\mu}^{1}\right) Z_{1}\left(x_{\mu}^{1}\right) \\
& +\frac{1}{2} \int_{x_{\mu}^{1}, x_{\mu}^{2}} \lambda\left(x_{\mu}^{1}\right) \lambda\left(x_{\mu}^{2}\right) Z_{2}\left(x_{\mu}^{1}, x_{\mu}^{1},\right)+\ldots
\end{aligned}
$$

where $Z_{0}$ describes a model where no hedgehogs are allowed. It can be shown (see Ref. [33]a) that due to the Berry phase terms on the square lattice, the contribution $Z_{1}\left(x_{\mu}^{1}\right)$ from single hedgehog event is oscillatory in space-time and thus rapidly vanishing upon integration over $x_{\mu}^{1}$ - it is therefore strongly "irrelevant". The same is true for $Z_{2}$ and $Z_{3}$, and the first non-vanishing contribution is from quadruple-hedgehog events $\left(\lambda_{4} \sim \lambda^{4}\right)$ corresponding to skyrmion number changes of \pm 4 . In powers of the one-hedgehog fugacity, this is already a "rare" event, as it requires four hedgehog events acting at the space-time point. It is compellingly argued in Ref. [32] that $\lambda_{4}$ is also irrelevant in a renormalization group $(\mathrm{RG})$ analysis right at the critical point. Therefore, hedgehogs are completely absent, in a RG sense, at the phase transition.

Now what is the role of the hedgehogs in the VBS? Whereas they are completely irrelevant in the Néel state, hedgehogs turn out to be crucial for the onset of VBS order. It can be rigorously shown [34] that the skyrmion creation operator, identified with the presence of a hedgehog, is equal to the VBS order parameter $\psi_{\text {VBS }}$ up to constant (see Ref.[32]b for an intuitive derivation). This essentially means that when hedgehogs proliferate, they do so in such a way that the spins pair into singlets and form a valence bond solid. This is again due to Berry phase effects. This dual property of hedgehogs - simultaneously destroying Néel and creating VBS orders - cannot be captured within a standard Landau analysis. The emergence of VBS order is moreover in agreement with Hastings' theorem which precludes a conventional gapped paramagnet.

So, the non-linear sigma model, supplemented with an analysis of the role of hedgehogs, contains in a way all the physics of both Néel and VBS phases. However, it is not possible to build a Landau theory out of it to describe the transition between both states, because hedgehogs are absent right at the critical point as we argued above.

\subsection{Gauge theory description}

We now consider an alternate formulation which enables us to glean more insight into the physics of the deconfined quantum critical point. Once again, 
gauge theory will provide the natural framework. We first rewrite the Néel vector in terms of spinor fields $z_{\alpha}$, where $\alpha=\uparrow, \downarrow$,

$\tilde{\mathbf{n}}=z_{\alpha}^{\dagger} \sigma_{\alpha, \beta} z_{\beta}$,

where $\sigma$ is the vector of Pauli matrices and the sum over double indices is implicit. Also, the normalization $|\tilde{\mathbf{n}}|^{2}=1$ requires $\left|z_{\downarrow}\right|^{2}+\left|z_{\uparrow}\right|^{2}=1$. This is the so-called $C P^{1}$ representation of the $S U(2)$ algebra. The spinors $z_{\alpha}$ can roughly be interpreted as the spinon fields. Eq. 15 simply means that we need two of these $S=1 / 2$ objects to get the $S=1$ spin-flip Néel field.

By definition, the spinor fields have a local $U(1)$ gauge redundancy - if we multiply $z$ by some phase factor $\exp \left(i \gamma\left(x_{\mu}\right)\right), \tilde{\mathbf{n}}$ stays unchanged. The spinons are therefore coupled to a $U(1)$ gauge field $a_{\mu}=\Im\left(z^{\dagger} \partial_{\mu} z\right)$. The non-linear sigma model action without Berry term can therefore equivalently be written as

$S_{\sigma}=\frac{1}{g} \int d x_{\mu}\left|\left(\partial_{\mu}-i a_{\mu}\right) z_{\alpha}\right|^{2}$.

Where are the hedgehogs in this formulation? Within the $C P^{1}$ representation, the skyrmion number $Q$ can be shown to be simply related to the flux of the gauge field

$Q=\frac{1}{2 \pi} \int d x d y\left(\partial_{x} a_{y}-\partial_{y} a_{x}\right)$.

A skyrmion is interpreted as a flux charge, and the hedgehog which changes this number is therefore a monopole in the gauge flux. A monopole event is sometimes called an instanton. Precisely at the critical point, the hedgehogs/monopoles have been argued to be irrelevant and their absence gives rise to a supplementary conservation law: the gauge flux is a conserved quantity. In absence of monopoles, a gauge theory is said to be noncompact. Thus a non-compact $C P^{1}$ gauge theory is a likely candidate to describe the physics of the critical point, since it incorporates from the very beginning both the spin $1 / 2$ and the absence of hedgehogs. A Landau theory in terms of both order parameters of the Néel and VBS phases does not incorporate this crucial feature, and thus also misses the important fact that the spinons, although absent in both phases, are the good "emerging" de- grees of freedom to describe the system at the critical point.

\subsection{Non-compact $C P^{1}$ theory and phase diagram}

With these arguments in hand, the authors of Ref. [32] now argue that the Néel-VBS quantum critical point can be described by a non-compact $C P^{1}$ gauge theory with the following Lagrangian

$$
\begin{aligned}
L_{\mathrm{NCCP} 1}= & \left|\left(\partial_{\mu}-i a_{\mu}\right) z_{\alpha}\right|^{2}+r|z|^{2}+u\left(|z|^{2}\right)^{2} \\
& +\kappa\left(\epsilon_{\mu \nu \lambda} \partial_{\nu} a_{\lambda}\right)^{2},
\end{aligned}
$$

where $\epsilon_{\mu \nu \lambda}$ is the antisymmetric tensor, and we recognize a magnetic-like energy in the last term. This is a Landau-like expansion, but in term of the spinon fields, which can admit a second-order phase transition, at least within a mean-field analysis. Specifically, for $r<0$, the model describes the Néel state, since the spinon field acquires a nonzero expectation value $\left\langle z_{\alpha}\right\rangle \neq 0$ and thus $\langle\tilde{\mathbf{n}}\rangle=$ $\left\langle z_{\alpha}^{\dagger}\right\rangle \sigma_{\alpha, \beta}\left\langle z_{\alpha}\right\rangle \neq 0$. For $r>0,\left\langle z_{\alpha}\right\rangle=\langle\tilde{\mathbf{n}}\rangle=0$, and we have a paramagnetic state, also with no hedgehogs. This hedgehog-free state is not an ordinary gapped quantum paramagnet. Rather, it turns out to be a critical $U(1)$ spin liquid, as described in Sec. 4. For $r=0$, we have a second-order transition between these two states: therefore the NéelVBS critical point is governed by the physics of a Néel- $U(1)$ spin liquid transition.

How can we gain intuition about this new phase transition? Refs. [32,35] argue that it can be described by a simpler classical model: a $3 \mathrm{~d}$ Heisenberg $O(3)$ model with no hedgehog topological defects. Such a model is hard to define analytically; it corresponds to the notation $Z_{0}$ in Eq. (14). However on a computer, a Monte Carlo calculation can be performed by simulating a classical $O(3)$ magnet and rejecting "by hand" all configurations which have a hedgehog. Such a calculation was performed [35] and a second order phase transition was found, between the ferromagnetic state and the no-hedgehog paramagnet, with a different set of critical exponents than if one allows free hedgehogs to exist. These calculations showed that a description of the transition can be given in terms of the spinon fields as in Eq. (18), and not in terms of the Néel field. The observed transition is argued to 


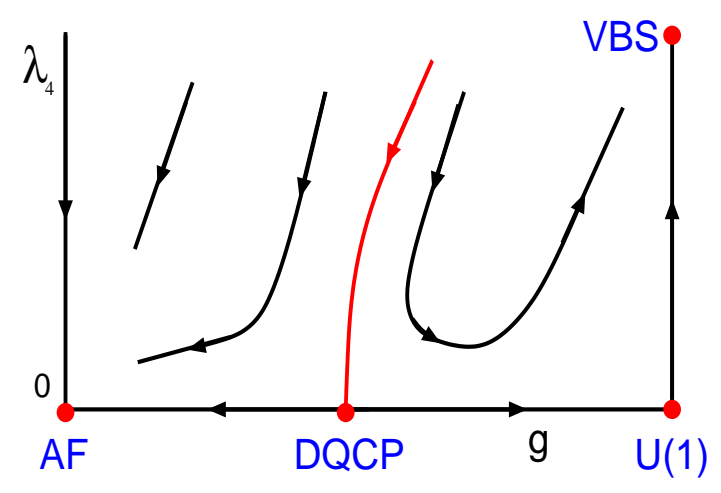

Fig. 14. Renormalization-group flow phase diagram with parameters $\lambda_{4}$ (the quadruple-hedgehog fugacity) and $g$ (the coupling strength that drives the transition). Four fixed points are visible (see text): the Néel (denoted AF), the VBS, the $U(1)$ spin liquid ( $U(1) \mathrm{SL}$ ) and the deconfined quantum critical point (DQCP).

be in the same universality class as the Néel-VBS $2 d$ quantum phase transition.

The "RG flow" phase diagram in terms of the fugacity of the quadruple-hedgehog event accounts for all the physical phases involved in the above discussion (see Fig. 14). The flow admits four fixed points. The first point is the antiferromagnetic Néel phase, where the fugacity renormalizes to zero, since the hedgehogs are too costly, and the VBS phase where hedgehogs proliferate, since they create the VBS order. Then a novel deconfined quantum critical point is present, where 4-hedgehogs events have been argued to be irrelevant. Finally, the phase diagram admits a $U(1)$ spin liquid point, which can be argued on general grounds [36] to be unstable with respect to the insertion of hedgehogs events. The deconfined quantum critical point might be hard to observe in numerical calculations, because in a lattice model the "bare" $\lambda_{4}$ is in general non-zero. However, there are many experimental predictions that can be made based on such a theory, in order to be able to detect deconfined criticality in the laboratory. We refer the interested reader to references [32] to find these predictions and to have a complete overview of deconfined quantum criticality. Other pedagogical introductions to this topic are also available [37].

\section{Conclusion}

We have discussed how "exotic" behaviour can arise in condensed matter systems which have strong interactions between electrons. Mott insulators with one electron per unit cell are intrinsically strongly interacting, and a good place to look for paradigm shifting phenomena. In particular, if no symmetry is broken, the ground-state is guaranteed to be an exotic "spin liquid". We have discussed two classes of spin liquids: topological and critical. The former exhibit topological order and gapped excitations with quantum number fractionalization. The latter display power-law correlations in the ground state and gapless excitations. In both cases, gauge theory offers a simple way to characterize these spin liquids and their excitations, and to encode the statistical interactions between them. Duality transformations also let us gain understanding of these novel quantum phases, which in some cases can be probed experimentally, as in the spin $1 / 2$ compound $\mathrm{Cs}_{2} \mathrm{CuCl}_{4}$.

We have also explored in the last section paths beyond Landau's theory of phase transitions through the example of the deconfined quantum critical point between a Néel and a Valence Bond Solid state in two dimensions. We reviewed the recent theoretical work of Ref. [32] that proposes a continuous transition between these phases, with emerging deconfined spinon excitations right at the critical point.

These recent developments hopefully convey the current excitement in the field of strongly interacting quantum condensed physics. The specific $Z_{2}$ and $U(1)$ spin liquids that were discussed above surely represent only the tip of a large iceberg consisting of many other exotic states of matters that can arise, especially in doped systems. But much hard work is needed to further understand and (hopefully!) detect such phases in the laboratory.

\section{Acknowledgments}

We first would like to thank the organizers of FPSPXI for the wonderful summer school and the enjoyable atmosphere. We would also like to thank 
Radu Coldea for kindly letting us reproduce the experimental data of Fig. 8. M.P.A.F. wishes to thank his many wonderful collaborators over the past ten years (you know who you are!) as well as numerous other friends and colleagues for many, many stimulating interactions (and for helping to keep him from straying too far afield into the hinterlands of exotica) and acknowledges the National Science Foundation for generous support through grants PHY-9907949 and DMR-0210790. F.A. would also like to warmly thank Grégoire Misguich for many discussions and insightful comments on the manuscript. A.M.W. wishes to thank Łukasz Cywiński for critical reading of the manuscript and the National Science Foundation through grants DMR0409937, PHY0216576 and PHY0225630 for support.

References

[1] See e.g. D. Pines and Ph. Nozières, The Theory of Quantum Liquids, Vol 1., Benjamin, New York (1966); L. D. Landau and E. M. Lifshitz, Statistical Physics, Pergamon, New York (1980) 3rd ed.

[2] For a review see e.g. S. Das Sarma and A. Pinczuk (ed.), Perspectives in Quantum Hall Effects, Wiley, New York (1996).

[3] L.D. Faddeev and L.A. Takhtajan, Phys. Lett. A 85, 375 (1981).

[4] For a recent review see e.g. M.R. Norman and C. Pépin, Rep. Prog. Phys. 661547 (2003).

[5] See e.g. G. R. Stewart, Rev. Mod. Phys. 73, 797 (2001); P. Coleman et al., J. Phys. Cond. Mat. 13, R723 (2001).

[6] See e.g. S. Maekawa et al., Physics of Transition Metal Oxides, Springer, Berlin (2004).

[7] M.B. Hastings, Phys. Rev. B 69, 104431 (2004).

[8] E.H. Lieb, T.D. Shultz and D.C. Mattis, Ann. Phys. (N.Y.) 16, 407 (1961).

[9] P.W. Anderson, Science 237, 1196 (1987).

[10] R. Moessner and S.L. Sondhi, Phys. Rev. Lett. 86, 1881 (2001); ibid, Phys. Rev. B 68, 184512 (2003); G. Misguich, D. Serban and V. Pasquier, Phys. Rev. Lett. 89, 137202 (2002).

[11] A.Y. Kitaev, Ann. Phys. 303, 2 (2003); C. Nayak and K. Shtengel, Phys. Rev. B 64, 064422 (2001); X.-G. Wen, Phys. Rev. Lett. 90, 016803 (2003).
[12] O.I. Motrunich and T. Senthil, Phys. Rev. Lett. 89, 277004 (2002); B. Douçot, M.V. Feigel'man, and L.B. Ioffe, Phys. Rev. Lett. 90, 107003 (2003).

[13] S. Fujimoto, Phys. Rev. B 72, 024429 (2005); K.S. Raman, R. Moessner and S.L. Sondhi, ibid. 72, 064413 (2005).

[14] L. Balents, M.P.A. Fisher and S.M. Girvin, Phys. Rev. B 65, 224412 (2002); D.N. Sheng and L. Balents, Phys. Rev. Lett. 94, 146805 (2005).

[15] A.Y. Kitaev, cond-mat/0506438 M. Freedman, C. Nayak and K. Shtengel, Phys. Rev. Lett. 94, 066401 (2005).

[16] L. B. Ioffe et al., Nature 415, 503 (2002).

[17] T. Senthil and M.P.A. Fisher, Phys. Rev. Lett. 86, 292 (2001).

[18] D.A. Bonn et al., Nature 414, 887 (2001).

[19] J.B. Kogut, Rev. Mod. Phys. 51, 659 (1979).

[20] T. Senthil and M.P.A. Fisher, Phys. Rev. B 63, 134521 (2001).

[21] X.-G. Wen, Quantum Field Theory of Many-Body Systems, Oxford University Press (2004).

[22] M. Hermele, T. Senthil and M.P.A. Fisher, Phys. Rev. B 72, 104404 (2005).

[23] G. Misguich and C. Lhuillier, in Frustrated spin systems, H. T. Diep ed., World-Scientific (2005) (also available as cond-mat/0310405).

[24] Y. Shimizu et al., Phys. Rev. Lett. 91, 107001 (2003).

[25] R. Coldea et al., Phys. Rev. Lett. 86, 1335 (2001); R. Coldea, D.A. Tennant and Z. Tylczynski, Phys. Rev. B 68, 134424 (2003); R. Coldea et al., Phys. Rev. Lett. 88, 137203, (2002); Y. Tokiwa et al (to be published).

[26] J. Alicea, O.I. Motrunich and M.P.A. Fisher, cond-mat/0508536 J. Alicea et al., Phys. Rev. B 72, 064407 (2005).

[27] E. Fradkin, Phys. Rev. Lett. 63, 322 (1989).

[28] M.P.A Fisher and D.H. Lee, Phys. Rev. B 39, 2756 (1989).

[29] M. Bocquet et al., Phys. Rev. B 64, 094425 (2001).

[30] Y. Zhou and X.-G. Wen, cond-mat/0210662

[31] S.V. Isakov, T. Senthil and Y.B. Kim, cond-mat/0503241

[32] T. Senthil et al., Science 303, 1490 (2004); ibid, Phys. Rev. B 70, 144407 (2004).

[33] F.D.M Haldane, Phys. Rev. Lett. 61, 1029 (1988); S. Chakravarty, B.I. Halperin and D.R. Nelson, Phys. Rev. B 39, 2344 (1989).

[34] N. Read and S. Sachdev, Phys. Rev. Lett. 62, 1694 (1989); ibid, Phys. Rev. B 42, 4568 (1990). 
[35] O.I. Motrunich and A. Vishwanath, Phys. Rev. B 70, 075104 (2004). In this work, local pairs of hedgehogs are actually allowed.

[36] See A.M. Polyakov, Gauge fields and strings, Hardwood Academic Publishers (1987).

[37] T. Senthil et al., J. Phys. Soc. Jpn. Suppl. 74, 1 (2005) (also available as cond-mat/0404718); T. Senthil, cond-mat/0411275 